\title{
El debate realismo vs. antirrealismo nomológicos y la inferencia a la mejor explicación*
}

\author{
Bruno Borge \\ Universidad de Buenos Aires - ANPCyT
}

Roberto Azar

Universidad de Buenos Aires

\begin{abstract}
Resumen: La disputa entre realistas y antirrealistas nomológicos se ha plasmado en la formulación de diversos argumentos y contraargumentos que alcanzan tópicos tan heterogéneos como la modalidad, la inducción y la misma práctica científica. En ese marco es frecuente tomar al principal argumento realista, el llamado argumento nomológico, por una instancia de la inferencia a la mejor explicación, mientras que el antirrealismo nomológico se considera una alternativa escéptica respecto de las leyes naturales fundamentada por razones independientes. El presente trabajo propone revisar esa imagen del debate realismo vs. antirrealismo nomológicos a la luz de lo que, consideramos, es una adecuada distinción entre abducción e inferencia a la mejor explicación.
\end{abstract}

Palabras clave: realismo nomológico; leyes naturales; leyes científicas; inferencia a la mejor explicación; abducción

\begin{abstract}
The Nomological Realism vs. Antirealism Debate and the Inference to the Best Explanation". The dispute between nomological realists and anti-realists has been reflected in the formulation of various arguments and counterarguments that reach topics as diverse as modality, induction and the very scientific practice. In this context it is common to take the main realist argument -the nomological argument-for an instance of Inference to the Best Explanation, while Nomological Anti-realism is considered a skeptical alternative concerning natural laws, sustained by independent reasons. This paper aims to review that image of the Nomological Realism vs. Anti-realism debate in light of what we believe is an appropriate distinction between abduction and Inference to the Best Explanation. Keywords: Nomological Realism; Natural Laws; Scientific Laws; Inference to the Best Explanation; Abduction
\end{abstract}

\footnotetext{
*Una versión preliminar de este trabajo fue presentada en el marco del II Congreso de la Sociedad Filosófica del Uruguay, realizado en la ciudad de Montevideo entre el 11 y el 16 de agosto de 2014. Agradecemos a la audiencia por sus valiosos comentarios y observaciones.
} 


\section{Introducción}

La cuestión de las leyes de la naturaleza ha sido un tópico ineludible desde los orígenes de la filosofia de la ciencia. Pese a ello, esta cuestión no ha recibido un tratamiento más que lateral o fragmentario hasta fines del siglo pasado. Desde entonces, el análisis sistemático de esta problemática ha dado lugar a dos polos que definen el marco del debate contemporáneo. Por una parte, para el Realismo Nomológico (RN) las regularidades y orden del mundo que permiten la formulación de las teorias científicas son adecuadamente explicados por la existencia de leyes naturales que dotan de algún tipo de necesidad al comportamiento regular de los fenómenos. Por su parte, quienes suscriben un Antirrealismo Nomológico (AN) -sea porque consideran que debemos seguir a Hume en su agnosticismo acerca de la necesidad natural, sea porque señalan que un compromiso con tal necesidad no implica uno con las leyes-sostienen que la regularidad de la naturaleza no nos compele a postular entidades tales como las leyes. Pese a estas divergencias, quienes participan en la polemica han aceptado ampliamente que el principal argumento a favor del RN, el llamado argumento nomológico ${ }^{1}$, constituye una instancia de lo que suele denominarse inferencia a la mejor explicación (IME), mientras que las distintas variantes de la alternativa antirrealista se consideran sustentadas por argumentos independientes. El argumento nomológico ha sido presentado de maneras diversas, sin embargo, su estrategia general consiste en postular a las leyes naturales como la mejor explicación de las regularidades. En el presente trabajo nos proponemos emprender una reconstrucción crítica de la problemática de las leyes naturales a fin de mostrar que, bajo una adecuada caracterización de la IME, el debate mismo acerca de las leyes, en todas sus inflexiones, constituye una instancia de esta forma de inferencia. Para ello, en la sección 2, procuramos trazar un mapa de la disputa Realismo vs. Antirrealismo Nomológicos priorizando un enfoque conceptual que permita, sin soslayar la importancia de las contribuciones de los actores más salientes de la polémica, brindar una caracterización precisa de las posiciones en juego y de sus presupuestos metafísicos. En la sección 3 exponemos el argumento nomológico, dando cuenta

\footnotetext{
1 Para una problematización del argumento nomológico, cf. Azar, R.M., "Realismo nomológico, una forma de nomo-teísmo", en: Ideas y Valores, v. LXV, 161 (2016), pp. 127-137.
}

ARETÉ Revista de Filosofia, vol. XXVIII, N², 2016 / ISSN 1016-913X 
tanto de sus diversas formulaciones como de la estructura común que le ha valido su identificación con la IME. En la sección 4 emprendemos un análisis histórico-conceptual de esta forma de inferencia a fin de fundamentar nuestra primera tesis, a saber, que la muy difundida identificación entre la abducción y la IME es errónea. En la sección 5 conjugamos los resultados precedentes exponiendo la tesis principal de nuestro trabajo: la discusión respecto del estatus de las leyes naturales constituye una instancia de la IME, mientras que el argumento nomológico constituye solo uno de sus momentos. Para finalizar, consignamos en la sección 6 las conclusiones de nuestro trabajo.

\section{La cuestión metafisica acerca de las leyes de la naturaleza}

La bien conocida distinción entre leyes científicas y generalizaciones accidentales monopolizó en buena medida las primeras discusiones en torno a la cuestión de las leyes. Se trataba entonces, a pesar de las múltiples inflexiones de la discusión, de respaldar conceptualmente la diferencia intuitiva que existe entre correlaciones necesarias entre propiedades (por ejemplo entre "ser un metal" y "dilatarse con el calor") y correlaciones factualmente constantes, pero contingentes (por ejemplo entre "ser una moneda de 50 centavos" y "estar en el bolsillo de Juan"). Este acercamiento tendia a soslayar las cuestiones metafisicas asociadas a la necesidad natural en favor de análisis semánticos y morfológicos cuyo objeto era identificar ciertas condiciones necesarias y suficientes que cierto enunciado debía presentar para ser considerado una ley. En esas disquisiciones, aspectos vinculados a la formulación de enunciados generales por parte de la ciencia se solaparon e incluso confundieron con las cuestiones ontológicas que abren la pregunta acerca del fundamento de lo regularidad natural; es decir, se tendía a tratar difusamente la distinción entre ley científica y ley de la naturaleza, clave para el desarrollo del debate contemporáneo. Ese desarrollo estuvo acompañado de un cambio paulatino pero radical en el foco de los argumentos. La preocupación por los aspectos lingüísticos, esto es, por las leyes científicas, fue cediendo terreno a las discusiones metafísicas acerca de las leyes de la naturaleza. Hay muchas maneras de esbozar un mapa del debate, tal como ha tenido lugar en las últimas décadas. Un recorrido histórico conceptual debería incluir sin dudas una reseña detallada de las posiciones de Lewis ${ }^{2}$,

2 Cf. Lewis, D., "New Work for a Theory of Universals", en: Australasian Journal of Philosophy, v. LXI (1983), pp. 343-377.

ARETÉ Revista de Filosofía, vol. XXVIII, N² 2, 2016 / ISSN 1016-913X 
Armstrong ${ }^{3}$, Carroll ${ }^{4}$ y Mumford ${ }^{5}$, entre muchas otras. A fines expositivos, sin embargo, preferimos limitarnos a brindar una imagen primordialmente conceptual de la cuestión, que permita hacer patentes los presupuestos metafisicos que sustentan las posiciones en juego, encausando en esa estrategia expositiva las referencias a las defensas que diferentes filósofos ensayaron de cada una de ellas.

Así es, entonces, como elegimos contar esta historia. Imaginemos tres pequeños mundos posibles: A, B y C. Los tres son idénticos en su composición: cada uno contiene un número $n$ de objetos, digamos, figuras geométricas, que pueden instanciar algunas propiedades, entre ellas, ciertos colores. Los detalles son irrelevantes más allá de dos descripciones básicas: (i) A, B y C son empíricamente indistinguibles, y (ii) en ellos ocurre que todos los círculos son azules, llamemos $p$ a la proposición que describe ese hecho ${ }^{6}$. Lo que diferencia a estos mundos no son el tipo de entidades y propiedades concretas que los pueblan, sino su naturaleza metafisica. En el mundo A todos los círculos son azules accidentalmente, pues el hecho de que sean azules es un mero factum. Ello no implica, sin embargo, que en A hayan existido ni lleguen a existir alguna vez círculos no-azules; por el contrario, se supone aquí que para cualquier instante $t_{n}$ de la historia total de A resulta verdadero que $p$. Ahora bien, mientras que en A todos los círculos son azules de manera puramente contingente, en $\mathrm{B}$ lo son necesariamente. Creemos que cualquiera puede obtener una interesante información acerca de su filiación filosófica preguntándose si encuentra que existe en verdad una diferencia entre A y B, o se trata en realidad del mismo mundo. Sin embargo, lo que resulta relevante para delinear el debate que nos ocupa es que B no puede ser descrito como un mundo que contiene leyes. Echemos entonces una mirada a C. Este mundo es, por (i), empíricamente idéntico al resto; además incluye, al igual que $\mathrm{B}$, conexiones necesarias. Pero eso no es todo, pues este mundo incluye además un rasgo extra, un tipo diferente de entidades del que B carece: leyes naturales que soportan la necesidad natural. ¿En qué consisten exactamente

3 Cf. Armstrong, D., What Is a Law of Nature?, Cambridge Mass.: Cambridge University Press, 1983.

4 Cf. Carroll, J., Laws of Nature, Cambridge Mass.: Cambridge University Press, 1994.

5 Cf. Mumford, S., Laws in Nature, Londres: Routledge, 2004.

6 La configuración de las ontologías de estos extraños mundos tiene como único propósito eliminar todo elemento que pueda desviar la atención del punto que pretendemos establecer; sin embargo, el mismo argumento puede aplicarse a nuestro mundo real y los mundos posibles equivalentes correspondientes.

ARETÉ Revista de Filosofía, vol. XXVIII, N² 2, 2016 / ISSN 1016-913X 
esas leyes? En el debate actual, dos son las opciones principales: para algunos (i.e. Armstrong ${ }^{7}$ ) las leyes son universales de segundo orden, relaciones de necesitación que vinculan universales de primer orden (en este caso, la propiedad de ser un círculo y la propiedad de ser azul); para otros (i.e. Ellis ${ }^{8}$ ) las leyes no son otra cosa que las propiedades esenciales de las clases naturales. Debe recodarse que A, B y C son mundos empíricamente equivalentes, ningún observador interno o externo notaría alguna diferencia en ellos en tanto sistemas de entidades considerados sincrónicamente, o respecto de su desarrollo en el tiempo. Sin embargo, si se aceptan sus matices metafísicos, se trata de tres mundos ontológicamente bien diferenciados.

La cuestión metafísica acerca de las leyes naturales ha tenido diversas aristas cuyo relevamiento resulta imposible a partir de este breve cuadro. No obstante, las principales distinciones que configuran el campo de debate pueden ser trazadas. Para el AN humeano, nuestro mundo es semejante a A, pero la ausencia de conexiones necesarias y el espíritu empirista heredado de Hume no deben entenderse como la ausencia de una propuesta metafísica. Por el contrario, para quienes sostienen posiciones en esta línea, el mundo está constituido por eventos discretos, cada uno de los cuales se autocontiene y es tanto ontológica como causalmente independiente del resto, tal como un mosaico cuyas piezas son estos eventos discretos y causalmente inertes, donde cada pieza es lo que es por sí misma. Muchas de esas piezas se presentan en relaciones regulares de semejanza y contigüidad espaciotemporal, pero fuera de ellas ninguna relación de necesidad las conecta. Eso es nuestro mundo, "una cosita y luego otra" 9 . La analogía es pertinente en un aspecto más: la independencia de cada una de las piezas no implica que no haya regularidades; por el contrario, con la dedicación suficiente podemos encontrar diversos y complejos patrones en la distribución de las piezas, podemos descubrir que toda pieza roja es contigua de una hexagonal, o que a cada grupo de piezas semejantes en color le sigue un número de piezas verdes determinable por alguna función, etcétera. El punto es que ninguna de esas regularidades identificadas por la investigación empírica está determinada por una necesidad interna (de una pieza implicando a otra) ni externa (del sistema total implicando alguna distribución). Las propuestas en esta línea suelen ser agrupadas bajo el nombre de teorias regularistas, pero a pesar de

\footnotetext{
7 Cf. Armstrong, D., o.c.

8 Cf. Ellis, B., Scientific Essentialism, Cambridge Mass.: Cambridge University Press, 2001.

9 Lewis, D., Philosophical Papers II, Oxford: Oxford University Press, 1986, p. ix.
} 
que en ocasiones el regularismo es presentado como una teoría acerca de las leye ${ }^{10}$, se trata en realidad de una teoria acerca de las regularidades o las conjunciones constantes en la naturaleza. Dicho de otro modo, según las consideraciones expuestas sobre la cuestión metafisica de las leyes, la teoría regularista de las mismas se enmarca, a pesar de su nombre, dentro del AN. En un sentido muy semejante se ha defendido la idea de que las leyes no se identifican con las regularidades, sino que supervienen a ellas, propuesta que suele denominarse superveniencia humeana de las leyes de la naturaleza ${ }^{11}$. Creemos que a dicha posición le caben las mismas consideraciones vertidas sobre las teorias puramente regularistas, aunque no desarrollaremos aquí este punto.

Para el AN no humeano, nuestro mundo es semejante a B, incluyendo conexiones necesarias en el elenco metafísico de la naturaleza, pero no entidades tales como leyes. La adhesión a esta forma de AN suele incluir la adopción de una metafísica disposicionalista ${ }^{12}$ : los poderes y disposiciones dotan al mundo de la efectividad causal que se requiere para fundamentar ontológicamente las regularidades. Según esta propuesta, las propiedades deben ser entendidas como clusters de poderes/disposiciones; así, que un particular sea F implica que también sea G no por medio de la acción externa de una entidad dudosa como la ley, sino por virtud de una necesidad inmanente a la constitución metafisica de F qua propiedad. Así son presentados los poderes, como pudiendo hacer todo el trabajo que harian las leyes con menor costo metafísico ${ }^{13}$.

Por último, el RN sostiene que nuestro mundo es en realidad alguna versión de $\mathrm{C}$, conteniendo no solo conexiones necesarias entre propiedades, sino leyes que las sustentan. Como hemos adelantado, la naturaleza de esas leyes ha sido esclarecida de modos diferentes. Para la concepción internalista las leyes existen, pero son internas a sus instancias, más precisamente, son

${ }_{10} C f$. Psillos, S., Causation and Explanation, Chesham: Acumen, 2002, p. 137.

$11 C f$. Beebee, H., "The Nongoverning Conception of Laws of Nature", en: Philosophy and Phenomenological Research, v. LXI (2000), pp. 571-594.

12 Cf. Cartwright, N., How the Laws of Physics Lie, Oxford: Clarendon Press, 1983; Mumford, S., o.c. Una revisión crítica de la posición de Mumford puede encontrarse en Borge, B., "Sobre la crítica de Mumford al realismo nomológico", en: Revista Internacional de Filosofía, v. XXXVIII, 3 (2015), pp. 59-80. http://dx.doi.org/10.1590/0100-6045.2015.V38N3.BB

${ }^{13}$ La economía en cuanto a compromisos ontológicos es en realidad solo una de las razones que se han esgrimido para abrazar el AN no humeano, a ella se suma una batería de argumentos dirigidos tanto contra el AN humeano como contra el RN. Mumford brinda un panorama bastante amplio de cómo se despliega esa estrategia argumental (cf. Ibid). 
las propiedades esenciales de las clases naturales. En palabras de Ellis: "todas las leyes de la naturaleza, desde las más generales... hasta las más específicas... derivan de las propiedades esenciales de los objetos y eventos que la constituyen, y deben regir en cualquier mundo de la misma clase natural que el nuestro" ${ }^{14}$. La cita tiene matices algo conflictivos, pero es leída como una afirmación de que, dado que el mundo tiene una estructura jerárquica de clases naturales, las relaciones de necesitación entre propiedades están dadas por su carácter esencial y constituyente de esas clases. Otra alternativa es adoptar la concepción externalista de las leyes defendida por Dretske ${ }^{15}$, Tooley ${ }^{16}$ y Armstrong ${ }^{17}$ (de aquí en adelante, DTA). La DTA sostiene que las leyes son un tipo de universal de segundo orden y, en esta geografia metafísica, las propiedades son entendidas como universales efectivamente instanciados que mantienen entre sí diversas relaciones de necesitación; esas relaciones de necesitación son las leyes. Si tener la propiedad F implica tener la propiedad $\mathrm{G}$, eso se debe a que $\mathrm{F}$ y $\mathrm{G}$ están vinculados en una relación $\mathrm{N}$ de necesidad tal que $\mathrm{N}(\mathrm{F}, \mathrm{G})$. Toda esta metafísica es inmanentista: no habria universales $\mathrm{F}$ ni G si no hubiese entidades en el mundo en las que $\mathrm{F}$ y G se instancien, como no habría relación $\mathrm{N}$ de necesitación (es decir, ley) si no hubiesen instancias en las que efectivamente $\mathrm{F}$ haga necesario a $\mathrm{G}$.

Esencias y universales son las dos formas principales que el RN ha dado a las leyes, y cada una de ellas ha sido sustentada por razones de diferente orden. No obstante, el hecho mismo de afirmar que nuestro mundo ha de ser como $\mathrm{M}_{3}$ se ha fundamentado a partir de que la existencia de las leyes parece ser la mejor explicación de la regularidad natural. No solo eso, ser la base de dicha regularidad otorga a las leyes un papel central en importantes tópicos como la inducción y la modalidad. Analicemos entonces con cuidado la estrategia argumental del RN.

\section{El argumento nomológico}

El discurso a favor de la existencia de las leyes de la naturaleza ha sido articulado en varios registros, desde referencias casi informales acerca de una legalidad natural que rige nuestro universo para que no funcione como

\footnotetext{
${ }^{14}$ Ellis, B., o.c., p. 4.

15 Cf. Dretske, F., "Laws of nature", en: Philosophy of Science, v. XLIV (1977), pp. 248-68.

${ }^{16}$ Cf. Tooley, M., "The Nature of Laws", en: Canadian Journal of Philosophy, v. VII (1977), pp. 667-698.

${ }^{17}$ Cf. Armstrong, D., o.c.
}

ARETÉ Revista de Filosofía, vol. XXVIII, N 2, 2016 / ISSN 1016-913X 
un caos indiferenciado hasta argumentos sistematizados que pretenden fundar el RN en bases estrictamente racionales. Sin embargo, en todo el arco que ambos extremos delimitan, la constante ha sido sostener que ciertas características de nuestro mundo parecieran quedar inexplicadas si no las suponemos fundadas en leyes de la naturaleza, o más directamente, que la existencia de dichas leyes constituye su mejor explicación. La especificación de dichas características ha variado según la presentación del argumento de la que se trate. Armstrong enfatiza entre ellas el éxito predictivo de la ciencia: "Si nuestras teorias no se acercasen a la verdad en muchos puntos, sería inexplicable que permitieran tantas predicciones exitosas. Los cálculos teóricos que pueden regresar a los hombres de la luna con una precisión de fracciones de segundo, dificilmente pueden ser meras fantasias. Podemos hacer una 'inferencia a la mejor explicación' del éxito predictivo de las teorias científicas contemporáneas a la conclusión de que estas teorias reflejan al menos algunas de las leyes de la naturaleza con una precisión aceptable"18. No solo el éxito de la empresa científica se explica a partir de la adecuación de las teorías a las leyes naturales, estas son también el fundamento de la inferencia inductiva: "Necesitamos una explicación de la racionalidad de la inducción. Mi propia explicación es la siguiente. El tipo de evidencia observacional que tenemos hace racional postular leyes que subyacen, y son en cierto sentido distintas de la evidencia observacional" 19 .

Argumentando en el mismo sentido pero con un espectro aun más amplio, algunos han sostenido que las leyes son la base y origen del orden del mundo, e incluso tal vez de su misma existencia: "Las leyes son importantes, un hecho que puede ser visto considerando que si no hubiera leyes el mundo, sería un lugar intrínsecamente caótico y aleatorio en el que la ciencia sería imposible. (Eso suponiendo que un mundo sin leyes pudiera existir, lo que me inclino a pensar que es dudoso, ya que ser cualquier tipo de cosa es estar sujeto a ciertas leyes)" 20 .

Lange procura mostrar que no solo el éxito predictivo de la ciencia, sino también su misma práctica carecerian de sentido si no se asumiera el $\mathrm{RN}$ : "Elaboro diversas intuiciones que sugieren que nuestra creencia en las leyes hace una diferencia respecto de nuestras creencias sobre algunas otras cuestiones...sostengo que la práctica cientifica incluye fenómenos tales que,

\footnotetext{
18 Armstrong, D., o.c., p. 6.

${ }^{19}$ Ibid., p. 52.

${ }^{20}$ Bird, A., Philosophy of Science, Londres: UCL Press, 1998, p. 26.

ARETÉ Revista de Filosofía, vol. XXVIII, N² 2, 2016 / ISSN 1016-913X
} 
para salvarlos, se debe utilizar el concepto de ley natural. Esta es la mejor manera que conozco para argumentar contra el escepticismo respecto de las leyes naturales"21.

Como queda de manifiesto, muchos han sido los rasgos de nuestro mundo invocados para argumentar a favor de la existencia de las leyes de la naturaleza: necesidad, orden, inducción, contrafactualidad, universalidad, éxito predictivo e incluso la misma facticidad de la práctica científica. Agrupar estos items implica conformar una clase sumamente heterogénea; sin embargo, todos sus elementos remiten de algún modo a un rasgo común: la regularidad. La regularidad de la naturaleza ha sido frecuentemente tomada por testimonio del orden del mundo, como base para el compromiso con modalidades de re, como fundamento de la inducción y la evaluación de enunciados contrafácticos $\mathrm{y}$, por las mismas o diversas razones, de la empresa cientifica y su éxito. Esta observación no diluye lo heterogéneo de las características que se invocan como base para los compromisos nomológicos, entre otras razones porque ninguna de ellas se vincula con la regularidad natural del mismo modo; pero permite, con todo, mostrar el núcleo de la estrategia que se ha utilizado en favor de dichos compromisos y ofrecer una versión resumida del argumento: la existencia de leyes naturales es la mejor explicación de la regularidad de nuestro mundo.

Si bien la regularidad natural puede sin dificultad ser tenida como el punto de partida para inferir la pertinencia del compromiso con las leyes en las distintas presentaciones del argumento nomológico, podría no parecer obvio que dicha estrategia argumental constituya una instancia de la IME. Esto, sin embargo, resulta explícito en el caso de Armstrong: "La inferencia a las leyes es un caso de la inferencia a la mejor explicación. (Si esta inferencia se rige por alguna norma de la lógica (no deductiva) es una cuestión sobre la que soy agnóstico). Si las leyes inferidas existen, entonces, por supuesto, implican predicciones condicionales sobre lo no observado ('si esto es un F, entonces será un G')"22.

Del mismo modo, y a pesar de que su exposición crítica conduce hacia el AN, Mumford presenta el argumento nomológico del siguiente modo: "Tomo la instancia central del argumento nomológico como una inferencia desde la regularidad u orden, a la existencia de leyes reales de la naturaleza como su

\footnotetext{
21 Lange, M., Natural Laws in Scientific Practice, Oxford: Oxford University Press, 2000, p. 4.

22 Armstrong, D., o.c., pp. 52-53.
} 
explicación y fundamento"23. Con mayor o menor fuerza, la idea de que las leyes constituyen la mejor explicación de la regularidad y, conversamente, de que esta permanecería inexplicada si no existiesen las leyes resuena en todas las versiones del argumento nomológico, incluso en las menos sistemáticas. Brindamos entonces, para terminar, una versión regimentada del argumento nomológico:

(R) El mundo natural es regular.

(L) La existencia de las leyes de la naturaleza fundamenta $\mathrm{R}$.

$\mathrm{L}$ es la mejor explicación disponible de R, por lo tanto, las leyes de la naturaleza existen.

Este esquema recoge las intuiciones comunes a buena parte de las presentaciones del argumento nomológico en la literatura especializada, a saber, que el argumento como tal es una IME, y que dicha inferencia va desde la regularidad de la naturaleza a la existencia de las leyes.

\section{Abducción e IME}

El término "inferencia a la mejor explicación" fue primeramente acuñado por Gilbert Harman ${ }^{24}$ para referirse a un mecanismo utilizado por los científicos en vistas de arribar a hipótesis capaces de explicar los fenómenos que investigan. Por otra parte, algunos años antes, el filósofo Charles Peirce había hablado de un procedimiento inferencial similar al que llamó "abducción"25.

Aunque tradicionalmente se ha tendido a identificar ambos tipos de inferencias, de modo que en la bibliografia especializada se suele hablar alternativamente de "inferencias a la mejor explicación" o de "inferencias abductivas", Harman se cuidó de no identificarlas: “La inferencia a la mejor explicación' corresponde aproximadamente a lo que otros han llamado 'abducción', 'el método de hipótesis', 'inferencia hipotética', 'el método de eliminación', 'inducción eliminativa' e 'inferencia teórica'. Yo prefiero mi propia terminología porque creo que evita la mayoría de las implicaciones engañosas de las terminologías alternativas". ${ }^{26}$

\footnotetext{
${ }^{23}$ Mumford, S., o.c., p. 72.

${ }^{24}$ Cf. Harman, G., "The Inference to the Best Explanation", en: The Philosophical Review, v. LXXIV, 1 (1965), pp. 88-95.

${ }^{25}$ Cf. Peirce, C.S., Collected Papers of Charles Sanders Peirce, Harstshorne, C. y otros (eds.), Cambridge Mass.: Harvard University Press, 1958.

26 Harman, G., o.c., pp. 88-89.
} 
Está claro que el introductor de la expresión "inferencia a la mejor explicación" fue absolutamente explícito acerca de la necesidad de diferenciar este proceso inferencial de otros con los cuales evidentemente se vincula de alguna forma. No obstante, muchos autores han insistido en difundir la idea según la cual la IME y la abducción constituyen el mismo tipo de inferencia. Para disipar esta confusión histórica, resultará menester rastrear el origen del término "abducción" y rescatar el verdadero sentido que Peirce le atribuyó a ese concepto. Ahora bien, la cuestión no es tan sencilla como a primera vista pudiera parecer, pues el autor ofreció al menos dos caracterizaciones diferentes, correspondientes a dos épocas distintas de su pensamiento. En efecto, podemos distinguir en el pensamiento de Peirce al menos dos fases que pasamos a describir brevemente: 1) en la fase temprana describió su teoría silogística, que puede ser vista como precursora del reciente uso de la abducción en la inteligencia artificial; 2) en la segunda etapa presentó su tardía teoría inferencial, en la cual la abducción representa la generación de hipótesis.

1) En los días de Peirce la lógica no estaba ni de cerca tan bien desarrollada como en la actualidad, y su primer intento de clasificar a los argumentos sigue a Aristóteles en el empleo de los silogismos.

El siguiente silogismo es conocido como BARBARA:

Todos los gatos negros traen mala suerte.

Los gatos que habitan esta casa son negros.

Por lo tanto, los gatos que habitan esta casa traen mala suerte.

La idea es que este argumento válido representa una instanciación particular de un esquema de razonamiento, y que cualquier instanciación alternativa representa otro argumento que es igualmente válido. Los silogismos deberian, luego, ser interpretados como esquemas argumentales.

Otros dos silogismos se obtienen a partir de BARBARA si intercambiamos la conclusión (o el resultado, como lo llama Peirce) o bien por la premisa mayor (la regla) o bien por la premisa menor (el caso).

Caso: Los gatos que habitan esta casa son negros.

Resultado: Los gatos que habitan esta casa traen mala suerte.

Regla: Todos los gatos negros traen mala suerte.

Regla: Todos los gatos negros traen mala suerte.

Resultado: Los gatos que habitan esta casa traen mala suerte.

Caso: Los gatos que habitan esta casa son negros. 
El primero de estos dos silogismos (inferencia de la regla a partir del caso y el resultado) puede ser reconocido como una generalización inductiva categórica, generalizando a partir de una muestra de gatos a la población total de gatos. La especie de inferencia ejemplificada por el segundo silogismo (inferencia del caso a partir de la regla y del resultado) es llamada por Peirce hipótesis -el término abducción solo es introducido en su teoría tardía-. Así, según la primera acepción de la palabra "abducción", esta constituye un tipo de razonamiento ampliativo, que tiene la forma de una falacia de afirmación del consecuente. Ahora bien, no es lícito concebir a la IME como una mera falacia de afirmación del consecuente, pues se trata de un proceso inferencial mucho más complejo que involucra no solo que al investigador se le ocurran hipótesis explicativamente apropiadas, sino además que dicho investigador disponga de algún mecanismo de comparación entre las hipótesis previamente propuestas.

Peirce, en esta primera etapa de su pensamiento, arriba a la siguiente clasificación de inferencias:

Inferencia $\rightarrow$ Deductiva o Analítica

$\rightarrow$ Sintética $\rightarrow$ Inducción

$\rightarrow$ Hipótesis (Abducción)

2) En su teoría tardía, Peirce abandonó la idea de una clasificación silogística de los razonamientos. En cambio, identificó a las tres formas de razonamiento -abducción, deducción e inducción- con las tres etapas de la investigación científica: generación de hipótesis, predicción y evaluación. El modelo subyacente de la investigación científica opera como sigue: cuando se enfrenta con un número de observaciones que busca explicar, el científico inventa una hipótesis inicial; luego, investiga qué otras consecuencias tendría esta hipótesis si fuera verdadera; finalmente, evalúa la medida en la cual estas consecuencias predichas acuerdan con la realidad. Peirce llama a la primera etapa, la invención de hipótesis que expliquen las observaciones iniciales, abducción; las predicciones son derivadas a partir de una hipótesis sugerida por deducción; y la credibilidad de la hipótesis es estimada a través de sus predicciones por inducción.

La abducción es ahora definida por Peirce como el proceso de formación de hipótesis explicativas a partir de una observación que requiere explicación. Este proceso no es algorítmico: "La sugerencia abductiva viene a nosotros como un destello. Es un acto de insight, aunque [se trata] de un insight extremadamente 
falible"27. En otro lugar Peirce describe a la abducción como una "capacidad para 'adivinar correctamente', un 'misterioso poder adivinador' que subyace a toda la investigación científica"28.

La forma inferencial es la siguiente:

El hecho sorprendente $\mathrm{C}$ es observado;

Pero si A fuera verdadera, C sería una cuestión corriente,

Por lo tanto, hay razón para sospechar que A es verdadera ${ }^{29}$

Acerca de C sabemos dos cosas: que es verdadero en el mundo real, y que es sorprendente (o inesperado).

"Si A fuera verdadera, C sería una cuestión corriente" es usualmente interpretado como "A implica lógicamente a C". Peirce llama A a una explicación de $\mathrm{C}$ o a una "hipótesis explicativa". Si esta es o no una noción apropiada de explicación permanece siendo un asunto de debate. Por ejemplo, autores como Console y Saitta ${ }^{30}$ coinciden en identificar a la explicación con la implicación, mientras que Josephson y Josephson ${ }^{31}$ argumentan en contra de esta tesis.

Además de ser explicativas, Peirce menciona dos condiciones más que deben cumplir las hipótesis abductivas: ellas deberian ser "capaces de verificación experimental" y deberían ser "económicas". Los factores económicos incluyen el costo de verificar la hipótesis y su valor intrínseco, entre otros aspectos $^{32}$. Es importante recalcar que los factores económicos son importantes en el proceso de conformación del lote de hipótesis explicativas surgido del conjunto de las que resultan lógicamente posibles. Precisamente por esta razón la abducción es a menudo llamada "inferencia a la mejor explicación"33. Sin embargo, creemos que no se trata de una condición suficiente para llevar a cabo semejante identificación, pues no debemos confundir los mecanismos por los cuales Peirce indica que una hipótesis puede surgir como una buena candidata para explicar un fenómeno dado con aquellos mecanismos -los propios de una IME- que permiten decidir cuál es la mejor hipótesis explicativa de un conjunto de hipótesis del que se dispone con anterioridad.

${ }^{27}$ Peirce, C.S., o.c., p. 5181.

${ }^{28}$ Ibid., p. 6530.

${ }^{29}$ Ibid., pp. 5188-5189.

${ }^{30}$ Cf. Console, L. y L. Saitta, "On the Relations between Abductive and Inductive Explanation", en: Flach, P. y A. Kakas (eds.), Topics in Abduction and Induction, Dordrecht: Kluwer Academic Press, 1998, pp. 145-159.

${ }^{31}$ Cf. Josephson, J. y S. Josephson, Abductive Inference: Computation, Philosophy, Technology, Cambridge Mass.: Cambridge University Press, 1994.

${ }^{32}$ Peirce, C.S., o.c., p. 7220.

${ }^{33}$ Cf. Lipton, P., Inference to the Best Explanation, Londres: Routledge, 1991. 
En resumen, según el segundo sentido de la palabra "abducción", esta ya no se vincula con un razonamiento ampliativo, sino que se trata específicamente del proceso (adecuado) de generación de hipótesis a partir de una observación intrigante que requiere explicación. En este caso, la abducción pertenecería exclusivamente al contexto de descubrimiento y de ninguna manera podríamos identificarla con la IME, pues esta última requiere al menos un paso adicional, en el que el investigador selecciona cuál es la mejor hipótesis que le ofrece un lote previamente constituido. Decidir si ese lote es bueno, malo o regular será tarea del investigador, pero la clave para no caer en la recurrente confusión consistente en identificar abducción e IME radica en comprender que se trata de procesos conceptualmente diferentes.

\section{IME y leyes naturales}

Como hemos visto en las secciones precedentes, el debate acerca de las leyes toma como punto de partida la constatación de que nuestro mundo presenta ciertas regularidades naturales. Dicha constatación constituye una evidencia observacional incuestionable que requiere una explicación o fundamento. En este marco, podemos relevar al menos tres respuestas posibles. En primer lugar, autores como Armstrong o Tooley insisten en afirmar que es innegable la existencia de leyes naturales sin las cuales conciben ininteligible la existencia de (R). Recuérdese que a esta posición se la denomina RN. Por otra parte, autores como Mumford o Cartwright -representantes del AN no humeano- perciben como absolutamente superfluo el recurso de apelar a entidades dudosas tales como las leyes. Por el contrario, estiman que la existencia de (R) es perfectamente comprensible con el solo reconocimiento de que el mundo presenta poderes o disposiciones, las cuales explican apropiadamente que un espejo de vidrio, pongamos por caso, se haga añicos cuando un aficionado de fútbol, lleno de ira ante la derrota de su equipo, lo golpea con un potente puñetazo, ya que el vidrio tiene una disposición natural a ser frágil tal que "si se lo golpea con cierta fuerza, entonces se rompe". No haría falta, según esta visión, imaginar la existencia de una relación de necesitación que se cumpla invariablemente entre las propiedades de primer orden de "ser un objeto de vidrio" y "ser frágil". Pues este mundo cargado de poderes hace todo el trabajo de las leyes con un costo metafísico mucho menor. Finalmente, podemos aludir a las posiciones regularistas o humeanas como proveyendo una respuesta alternativa a las dos que hemos reseñado. Si bien profundizaremos sobre este 
punto más abajo, adelantemos que tendemos a creer que el regularismo o AN humeano, en cualquiera de sus múltiples variantes, lo que hace en esencia es lo siguiente: proponer una metafísica deshinchada o no inflacionaria en la cual la regularidad es un mero factum indiscutible, casi una feliz coincidencia. Según estas visiones que privilegian una política económica austera en lo que se refiere a la población ontológica de la realidad, no se requiere postular nada más para lograr una apropiada comprensión del mundo natural que nos rodea. De hecho, en el extremo de esta posición, Swartz afirma que las coincidencias cósmicas son lo único en lo que es preciso creer $^{34}$.

En general, tanto realistas como antirrealistas acuerdan en sostener que el argumento nomológico empleado por los defensores del RN constituye una instancia de la IME, interpretada como una abducción. Nuestra tesis, sin embargo, enfoca la cuestión de un modo diferente, coherente con las distinciones que hemos trazado tanto en el terreno propio de la caracterización metafísica de las leyes, como en lo concerniente a la abducción e IME. Sostenemos que el debate acerca de las leyes naturales, considerado como una totalidad que incluye a todo el arco de posiciones encontradas, constituye una instancia de la IME. Dicha forma de inferencia, como se dijo, se ha asociado fuertemente a la estrategia emprendida por los realistas nomológicos, pero ello se debe en gran medida, según nuestra opinión, a la errónea identificación entre abducción e IME. Sentada esa distinción, la propuesta de cada una de las diferentes posiciones alternativas acerca del fundamento de la regularidad natural -incluida la del RN- ilustra el desarrollo del proceso abductivo por el cual se conforma el lote de hipótesis explicativas en juego, i.e., se trazan los limites de la disputa filosófica en torno a las leyes de la naturaleza. Ahora bien, la disputa en sí misma, el proceso en el cual cada contendiente da cuenta de las ventajas comparativas de su propuesta o impugna los argumentos de sus rivales a favor de otras hipótesis explicativas en danza, constituye el desarrollo de la fase selectiva a la que propiamente le cabe el rótulo de IME.

Un hecho que se hace patente en cualquier reconstrucción del debate RN vs. AN -lo que incluye, por supuesto, nuestra propia reconstrucción ensayada en la sección 2- es que la regularidad natural no es el punto de partida solo para los realistas nomológicos; por el contrario, es el factum del que tanto realistas como antirrealistas pretenden dar cuenta. La regularidad

\footnotetext{
${ }^{34}$ Cf. Swartz, N., The Concept of Physical Law, Cambridge Mass.: Cambridge University Press, 1985.
}

ARETÉ Revista de Filosofía, vol. XXVIII, Nº 2, 2016 / ISSN 1016-913X 
de la naturaleza juega aquí el papel de la evidencia que parece pedir una explicación. Ante ella, algunos (Ellis, por ejemplo) han visto a las propiedades esenciales de las clases naturales como su fundamento ontológico y explicativo: es porque la naturaleza tiene una estructura estable de clases esencialmente diferenciadas que las entidades que la constituyen se relacionan de modo regular. Otros, también dentro del bando realista (por ejemplo los defensores de la DTA) optaron por postular leyes que, vinculando de manera indisoluble ciertas propiedades consideradas como universales, explican las conjunciones constantes de sus instancias en la experiencia. Sin embargo, esta propuesta no es entendida siquiera por sus propios creadores como la única hipótesis capaz de explicar la regularidad; el mismo Armstrong es consciente de que otras alternativas pueden ser formuladas $y$, de hecho, puede pensarse que las anticipa lanzando un desafio: "Todavía podría preguntarse si la apelación a las leyes es realmente la mejor explicación de [la regularidad observable]. Podemos responder a ello con un reto: 'Produzca una mejor, o igualmente buena explicación'. Tal vez el reto pueda cumplirse, simplemente vamos a esperar y ver"35.

Por su parte, desde el flanco antirrealista, los humeanos ofrecen una pintura metafísica de unidades discretas y causalmente inertes, donde los patrones son descubiertos por quienes siguen el rastro de las conjunciones constantes; la regularidad en este marco es simplemente un factum desnudo del que la investigación empírica da cuenta. En el extremo de esta posición, encontramos la mencionada hipótesis de Swartz: la regularidad de la naturaleza no es más que una gran coincidencia cósmica ${ }^{36}$. Sin embargo, esto no debe confundirse con la ausencia de una explicación: una enorme casualidad es una hipótesis explicativa arriesgada que presupone profundos compromisos metafísicos. Pero esa no es la única respuesta dentro del antirrealismo. Mumford, que no cree en las casualidades, propone su propia explicación de la regularidad natural: poderes y disposiciones inmanentes a las propiedades que constituyen nuestro universo lo dotan de una dinámica causal intrínseca, que es regular en tanto dichos poderes guardan relaciones de implicación y exclusión inscritas en su misma naturaleza. No son ya las leyes, sino la misma constitución modal de las propiedades las que dan cuenta del comportamiento regular de los fenómenos. No obstante, al igual que Armstrong, Mumford inscribe su propuesta en el marco del debate como una hipótesis alternativa

35 Armstrong, D., o.c., p. 89.

${ }^{36}$ Swartz, N., o.c., p. 202.

ARETÉ Revista de Filosofía, vol. XXVIII, N² 2, 2016 / ISSN 1016-913X 
más, que tiene por tanto que probar su fuerza y conveniencia respecto de sus rivales: "Si las leyes no pueden hacer el trabajo para el que originalmente estaban destinadas, entonces resulta muy poco atractivo retenerlas. Pero la metafisica sin leyes que defiendo necesita más que eso. Además de mostrar que las leyes no pueden hacer el trabajo, es necesario mostrar que hay otra cosa diferente de ellas capaz de proveer lo que pensamos que las leyes podian darnos"37. Como se ve, Mumford es consciente de que la hipótesis explicativa que él prefiere no tiene por qué gozar de un privilegio especial, al menos si no se prioriza -como él lo hace- una explicación que logre dar cuenta de los mismos hechos con el menor costo metafísico posible.

Según nuestra lectura, estas líneas argumentales seguidas por algunos filósofos que participan activamente en la polémica da cuenta de que las distintas propuestas, tanto realistas como antirrealistas, pretenden robustecer a alguna de las hipótesis explicativas que el proceso abductivo ha dado como resultado, mostrando así que se trata de la mejor disponible a la hora de dar cuenta de la regularidad de la naturaleza.

\section{Conclusión}

El debate RN vs. AN es, sin duda, un complejo escenario que nuestra reconstrucción no consigue más que esbozar. Con todo, las principales posiciones y sus fundamentos han sido caracterizadas. Entre estos, prestamos especial atención al argumento nomológico, recorriendo sus diversas formulaciones para llegar a brindar una versión que rescate su sentido y tesis principales. Dada su usual vinculación con la IME, brindamos nuestras razones para sostener que esa forma de inferencia es erróneamente identificada con la abducción, cuando, a nuestro entender, se trata de procesos complementarios pero diferenciados. Con estas nociones procuramos mostrar cómo puede conjugarse una mirada global sobre la cuestión de las leyes con esa distinción. De acuerdo a nuestra reconstrucción de la polémica, tanto la adecuada caracterización de la IME como la valoración del sentido que los diversos argumentos han seguido en ese marco dan cuenta de que el debate acerca de las leyes, en su totalidad, puede ser adecuadamente entendido como una gran IME.

\footnotetext{
${ }^{37}$ Mumford, S., o.c., p. 159. 


\section{Bibliografia}

Armstrong, D., What Is a Law of Nature?, Cambridge Mass.: Cambridge University Press, 1983.

Azar, R.M., "Realismo nomológico, una forma de nomo-teísmo", en: Ideas y Valores, v. LXV, 161 (2016), pp. 127-137.

Beebee, H., "The Nongoverning Conception of Laws of Nature", en: Philosophy and Phenomenological Research, v. LXI (2000), pp. 571-594. http://dx.doi.org/10.2307/ 2653613

Bird, A., Philosophy of Science, Londres: UCL Press, 1998. http://dx.doi.org/10.4324/ 9780203165348

Borge, B., "Sobre la crítica de Mumford al realismo nomológico", en: Revista Internacional de Filosofia, v. XXXVIII, 3 (2015), pp. 59-80. http://dx.doi.org/10.1590/01006045.2015.V38N3.BB

Carroll, J., Laws of Nature, Cambridge Mass.: Cambridge University Press, 1994. http:// dx.doi.org/10.1017/CBO9780511619908

Cartwright, N., How the Laws of Physics Lie, Oxford: Clarendon Press, 1983. http:// dx.doi.org/10.1093/0198247044.001.0001

Console, L. y L. Saitta, "On the Relations between Abductive and Inductive Explanation", en: Flach, Py A. Kakas (eds.), Topics in Abduction and Induction, Dordrecht: Kluwer Academic Press, 1998, pp. 145-159.

Dretske, F., "Laws of nature", en: Philosophy of Science, v. XLIV (1977), pp. 248-68. http://dx.doi.org/10.1086/288741

Ellis, B., Scientific Essentialism, Cambridge Mass.: Cambridge University Press, 2001.

Harman, G., "The Inference to the Best Explanation", en: The Philosophical Review, v. LXXIV, 1 (1965), pp. 88-95. http://dx.doi.org/10.2307/2183532

Josephson, J. y S. Josephson, Abductive Inference: Computation, Philosophy, Technology, Cambridge Mass.: Cambridge University Press, 1994. http://dx.doi.org/10.1017/ CBO9780511530128

Lange, M., Natural Laws in Scientific Practice, Oxford: Oxford University Press, 2000.

Lewis, D., "New Work for a Theory of Universals", en: Australasian Journal of Philosophy, v. LXI (1983), pp. 343-377. http://dx.doi.org/10.1080/00048408312341131

Lewis, D., Philosophical Papers II, Oxford: Oxford University Press, 1986.

Lipton, P., Inference to the Best Explanation, Londres: Routledge, 1991.

Mumford, S., Laws in Nature, Londres: Routledge, 2004. http://dx.doi.org/10.4324/ 9780203458426

Peirce, C.S., Collected Papers of Charles Sanders Peirce, Harstshorne, C. y otros (eds.), Cambridge Mass.: Harvard University Press, 1958.

230 Pasillos, S., Causation and Explanation, Chesham: Acumen, 2002, p. 137.

Swartz, N., The Concept of Physical Law, Cambridge Mass.: Cambridge University Press, 1985.

Tooley, M., "The Nature of Laws", en: Canadian Journal of Philosophy, v. VII (1977), pp. 667-698. http://dx.doi.org/10.1080/00455091.1977.10716190

Recibido: $31 / 08 / 14$

Aceptado: 12/12/15

ARETÉ Revista de Filosofía, vol. XXVIII, N² 2, 2016 / ISSN 1016-913X 